\title{
Conservação e Digitalização do Registro Geomagnético Histórico do Observatório Magnético de Vassouras
}

Evelyn Paiva do Nascimento'; Ian Muzy Camarão Peixoto1; Rômulo Rodrigues de Oliveira1; Ana Luiza Muniz; Daniel R. Franco2; Gelvam A. Hartmann³; Marcelo B. Bianchi ${ }^{4}$; Ozana Hannesch'; Ana Cristina de Oliveira Garcia ${ }^{5}$; Alberto Faria dos Santos $^{2}$; Marcio Ferreira Rangel ${ }^{5}$; Amal Abdulmalek ${ }^{2}$; Highlander Halfeld de Aguiar6; Vanessa Alves da Costa ${ }^{7} ;$; Laisa da Fonseca Aguiar ${ }^{1}$; Etieli da Silva Santos ${ }^{2}$; Everaldo Pereira Frade ${ }^{5}$; André Wiermann ${ }^{2}$; Luci Meri Guimarães ${ }^{5}$; Helder Faria Ladeira 2; Vitor Bernardes; Andrés R.R. Papa²; Ricardo I.F. Trindade4; Fábio Pinto Vieira²; Kátia Jabinschek dos Reis Pinheiro²; Gabriel Brando Soares²; Elder Yokoyama ${ }^{8}$; José Roberto Barbosa ${ }^{4}$.

\footnotetext{
${ }^{1}$ Departamento de Geologia e Geofísica, Instituto de Geociências, Universidade Federal Fluminense (IGc-UFF).

2 Coordenação de Geofísica, Observatório Nacional (COGEO-ON/MCTIC).

3 Instituto de Geociências, Universidade Estadual de Campinas (IGc-UNICAMP).

4 Instituto de Astronomia, Geofísica e Ciências Atmosféricas, Universidade de São Paulo (IAG-USP).

${ }^{5}$ Coordenação de Documentação e Arquivo, Museu de Astronomia e Ciências Afins (CDA-MAST/MCTIC).

${ }^{6}$ Departamento de Ciências Tecnológicas e Exatas, Universidade de Vassouras.

${ }^{7}$ Laboratório de Engenharia e Exploração de Petróleo, Universidade Estadual do Norte Fluminense (LENEP/UENF).

8 Instituto de Geociências, Universidade de Brasília (IGc-UnB).
}

Copyright 2019, SBGf - Sociedade Brasileira de Geofísica

This paper was prepared for presentation during the $16^{\text {th }}$ International Congress of the Brazilian Geophysical Society held in Rio de Janeiro, Brazil, 19-22 August 2019.

Contents of this paper were reviewed by the Technical Committee of the $16^{\text {th }}$ International Congress of the Brazilian Geophysical Society and do not necessarily represent any position of the SBGf, its officers or members. Electronic reproduction or represent any position of the SBGt, its officers or members. Electronic reproduction

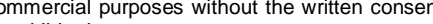
of the Brazilian Geophysical Society is prohibited.

\section{Abstract}

In this work, we present the first set of results provided by a joint effort of researchers and students of the National Observatory (ON/MCTIC), Institute of Astronomy, Geophysics and Atmospheric Sciences - University of São Paulo (IAG/USP), and Institute of Geosciences State University of Campinas (IGc-UNICAMP), aiming to provide the cataloguing, digitization and online availability of historical geomagnetic and seismographic datasets which were carried out throughout the 20th century. Such geophysical repository is available, respectively, by historical collections of magnetograms from the Vassouras Magnetic Observatory (state of Rio de Janeiro, Brazil) and seismograms from the IAG/USP. Our goal is to provide high-resolution digital records of these data, which will enable access to records of the major 20th century quakes in Brazil, as well as to a $~ 100 \mathrm{yr}$ record of the geomagnetic field for both the scientific community and the public in general.

\section{Introdução}

Nos últimos anos, a recuperação, catalogação e arquivamento de dados científicos históricos vêm ganhando importância na comunidade acadêmica internacional. As bases de dados históricos são provenientes das mais variadas vertentes científicas, que possibilitam, por exemplo, novas abordagens e revisões sobre resultados anteriormente obtidos. No âmbito da Geofísica, informações adquiridas por diferentes abordagens apresentam particularidades que devem ser devidamente consideradas. Como exemplo, os registros das variações do campo geomagnético eram adquiridos, até poucas décadas atrás, pela utilização de três sensores distintos (variógrafos), sendo cada um deles responsável pelo registro de uma das componentes do campo geomagnético. Para cada componente havia uma correção distinta (linha de base), que era registrada concomitantemente à variação temporal do campo geomagnético. Essas componentes eram gravadas em folhas de papel fotossensível - os chamados magnetogramas. Cada magnetograma contém o registro das componentes do campo geomagnético (componentes horizontal, vertical e declinação) durante o intervalo que vai da segunda metade de um determinado dia até o final da primeira metade do dia seguinte (Fig. 1).

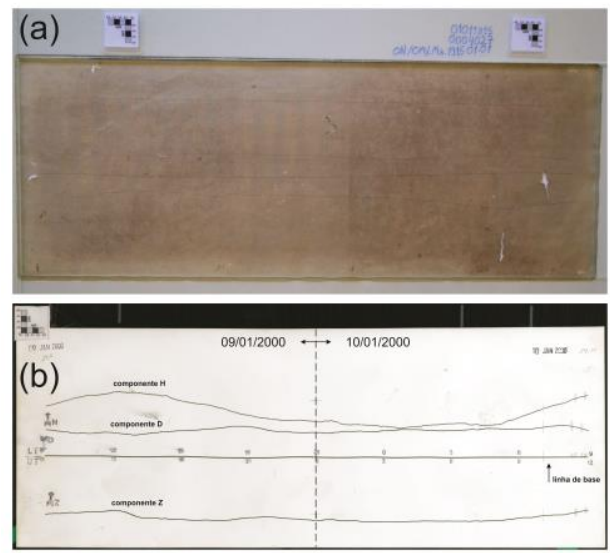

Figura 1: exemplos de magnetogramas do acervo geomagnético histórico do Observatório de Vassouras (OMV). (a) imagem do primeiro magnetograma obtido, datado de 01/01/1915; (b) exemplo de magnetograma, obtido entre os dias 09/01/2000 e 10/01/2000. 
Atualmente, com 0 advento de magnetômetros e sistemas de aquisição de dados associados, tal tipo de informação vem sendo gravada em mídia digital, em formato próprio para o processamento e interpretação. De forma equivalente, somente em meados da década de 1980, com o avanço e disponibilidade de microcomputadores, que registros sismográficos passaram a ser feitos de forma digital com correção de tempo pelo sistema GPS (garantindo uma confiabilidade de tempo da ordem de milissegundos), e não em papel, como feito nas décadas anteriores à década de 1980 .

No entanto, os registros magnéticos e sismográficos gravados anteriormente ao estabelecimento do formato digital pelo Observatório Nacional (ON/MCTIC) e pelo Instituto de Astronomia, Geofísica e Ciências Atmosféricas da Universidade de São Paulo (IAG/USP) vinham se perdendo pela decomposição natural dos registros em papel. Tal situação, que demandava urgência no sentido de se implementarem iniciativas para que se evite a perda completa das informações neles contidas, serviu de base para o estabelecimento de um projeto interinstitucional - envolvendo pesquisadores e estudantes de diversos institutos de pesquisa e universidades brasileiras -, visando a digitalização destes registros para a preservação das medidas geofísicas realizadas, bem como permitir o uso de novas metodologias para interpretação dos dados antigos em conjunto com os dados atuais - tarefa esta de grande importância tanto do ponto de vista científico como também do ponto de vista histórico.

De maneira geral, nosso objetivo é o de se catalogar, digitalizar e disponibilizar os seguintes acervos geofísicos históricos: (a) coleção de magnetogramas, livros de linha de base e de valor de escala, registrados pelos Observatórios Magnéticos de Vassouras (RJ) e de Tatuoca (PA) (sob supervisão da Coordenação de Geofísica do ON/MCTIC), para os períodos respectivamente compreendidos entre 1915 e 2007, e 1957 e 2007; e (b) coleção de sismogramas provenientes das estações sismográficas operadas pelo IAG/USP, no período compreendido entre 1976 e 1995. Para a disponibilização dos acervos, a equipe está desenvolvendo métodos para tratamento e recuperação da informação contida nas imagens obtidas em alta resolução destes documentos. O acesso aos dados provenientes do processamento planejado será subsequentemente viabilizado à comunidade científica nacional e internacional por meio de um portal online, especialmente desenvolvido para esse fim.

No presente trabalho, discutiremos a respeito dos procedimentos adotados e os mais recentes avanços sobre uma das principais etapas deste projeto, envolvendo o registro geomagnético do Observatório Magnético de Vassouras (OMV). Além de ser um dos maiores acervos científicos do país, contando com cerca de 33.000 documentos, o registro histórico geomagnético do OMV tem importância como testemunho sobre a história do desenvolvimento cientifico no Brasil, além de ser o primeiro conjunto de dados magnéticos relativo à uma das principais anomalias geomagnéticas - a Anomalia Magnética do Atlântico Sul (AMAS). O significado científico e histórico da execução desta fase representa o provimento de acesso, em alta resolução amostral, ao primeiro registro do campo geomagnético para a América do Sul obtido de forma sistemática (registrado em magnetogramas de maneira praticamente contínua entre 1915 e 2007), e permitirá estudar as séries históricas sobre a AMAS, que atualmente desempenha papel fundamental no controle do campo geomagnético global.

\section{Procedimentos e Métodos}

\section{Catalogação e higienização do acervo do OMV}

Para a execução da fase de aquisição de dados geomagnéticos históricos do OMV, foram tomados cuidados preliminares com relação à preparação dos documentos para o posterior transporte e registro de imagens em alta resolução, dado que se encontravam guardados de maneira inadequada nas dependências desta unidade do ON/MCTIC.

Tais procedimentos envolveram a higienização, catalogação e recondicionamento dos magnetogramas. Cada um dos cerca de 33.000 magnetogramas, e todos os livros de linha de base e valor de escala foram higienizados (remoção de poeira; Fig. 2-A e 2-B), reorganizados e codificados de acordo com normas estabelecidas pela equipe da Coordenação de Documentação e Arquivo do Museu de Astronomia e Ciências Afins (CDA-MAST). A codificação conta com três modelos de códigos, que foram estabelecidos pelas instituições participantes do projeto para identificação futura dos documentos (Fig. 2-C). Informações contidas no verso dos documentos e demais características vêm sendo registradas em um banco de metadados, disponível em http://www.dadosraros.iag.usp.br/acervo/.
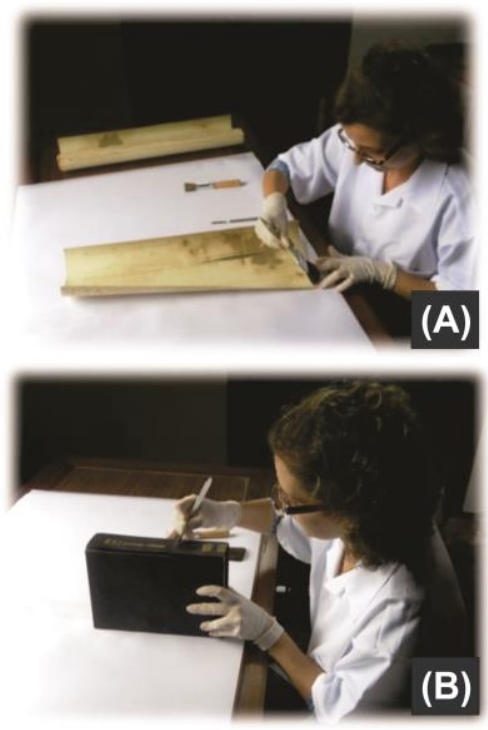

Figura 2: etapa de catalogação e higienização. (A) higienização de magnetograma; (B) higienização de livro de linha de base; (C) Codificação no verso de magnetograma, adquirido entre os dias 03 e 04 de janeiro de 1917.

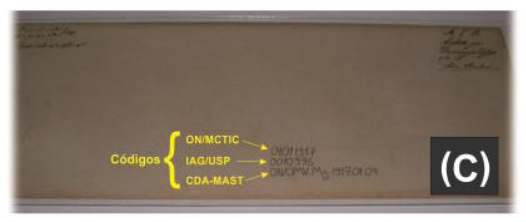




\section{Imageamento de salvaguarda}

Uma das precauções que foram tomadas durante esta fase foi a da aquisição de imagens de salvaguarda de todos os documentos do acervo geomagnético histórico, precedendo o transporte destes entre os campi do OMV e o de São Cristóvão (onde também se situa a sede do MAST). Este cuidado foi adotado pelo fato destes registros não possuírem duplicatas, o que implicaria, em caso de extravio, na perda definitiva das informações geomagnéticas originais contidas nos documentos históricos.

A aquisição destas imagens (Fig. 3) foi realizada pela utilização de um aparato envolvendo uma mesa com vidro antireflexo (abaixo do qual os magnetogramas eram posicionados), acoplada a um suporte para máquina fotográfica, acionada por um disparador. À cada tomada de imagem de magnetograma, uma escala graduada (cm) bidimensional foi associada, de maneira a permitir correções de distorção em processos de aquisição de dados geomagnéticos, que eventualmente fossem baseados em tal processo. As imagens obtidas foram armazenadas em arquivos backup, possibilitando então a liberação dos documentos correspondentes para o translado ao campus de São Cristóvão.

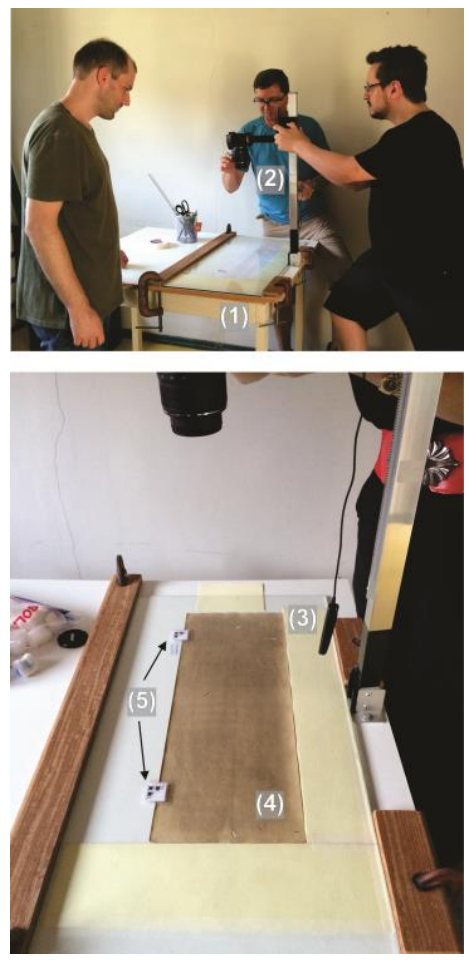

Figura 3: processo de aquisição de imagens de salvaguarda. (1) mesa com vidro antireflexo; (2) suporte para máquina fotográfica; (3) disparador do obturador da máquina fotográfica; (4)

magnetograma; (5) escala graduada $(\mathrm{cm})$ bidimensional.

\section{Imageamento em alta resolução}

Conforme discutido anteriormente, as caixas de magnetogramas (cada uma contendo um ano de medidas geomagnéticas) eram transportadas à sede principal do ON/MCTIC, situada na cidade do Rio de Janeiro, após a realização do imageamento de salvaguarda nas dependências do OMV, em Vassouras (RJ). De lá, são diretamente encaminhadas ao CDA-MAST para a realização de aquisição de imagens em scanners de alta performance Omniscan 12 (modelo OS12000), que permitem o rápido processamento de grandes volumes de dados provenientes do imageamento de documentos.

Esta etapa do trabalho vêm resultando na aquisição de imagens JPEG dos magnetogramas com resolução de 600 dpi, intensidade de 24 bits, dimensões de $12.000-$ 13.000 pixels (largura) / 5000 - 6000 pixels (altura), e que tem sido feito de tal forma que as linhas de base associadas às medições de campo se apresentem horizontalizadas (garantindo, assim, uma melhor precisão e acurácia no processo de aquisição de dados). À exemplo do imageamento de salvaguarda, as imagens obtidas por este processo sempre foram associadas a escalas graduadas bidimensionais, o que possibilita a conversão dos valores de cota extraídos das imagens para valores nas escalas das componentes geomagnéticas.

\section{Aquisição de dados digitais}

Para a aquisição dos dados geomagnéticos provenientes dos magnetogramas, as imagens em alta resolução são processadas para digitalização das informações contidas, através do software "Web Plot Digitizer" (https://automeris.io/WebPlotDigitizer) para que os valores atrelados às curvas de variação e linhas de base sejam extraídos em termos de posição na escala de pixels, e em função do tempo. Em seguida, tais dados são pré-processados, visando condicioná-los a um algoritmo, ainda em fase de desenvolvimento, e que possibilita a conversão de uma determinada curva de componente geomagnética, obtida na aquisição de dados digitais na escala de pixels, para a escala "real" desta componente geomagnética.

\section{Resultados e Conclusões}

No presente momento, já foram feitas imagens de salvaguarda para quase a totalidade dos documentos que compõem o acervo do Observatório Nacional de Vassouras, dos quais mais da metade já foram escaneados em alta resolução. A Tabela 1 abaixo apresenta os resultados alcançados até então por este projeto interinstitucional.

Tabela 1: Resultados alcançados pelo projeto.

\begin{tabular}{|c|c|}
\hline \multicolumn{2}{|l|}{ Imageamento de salvaguarda } \\
\hline Número de anos com imagens de salvaguarda $\left(^{*}\right)$ & 90 \\
\hline Percentual alcançado do acervo & $98,9 \%$ \\
\hline Total de magnetogramas (estimativa) & 33.215 \\
\hline \multicolumn{2}{|l|}{ Imageamento em alta resolução } \\
\hline Número de anos com imagens em alta resolução $\left(^{*}\right)$ & 47 \\
\hline Percentual alcançado do acervo & $51,6 \%$ \\
\hline Total de magnetogramas (estimativa) & 17.155 \\
\hline
\end{tabular}




\section{Conclusões}

Este esforço interinstitucional já possibilitou a preservação das informações contidas em praticamente todo o acervo geomagnético histórico do OMV - que, conforme discutido anteriormente, representa o primeiro conjunto de dados geofísicos obtidos de forma sistemática na América do Sul.

Recentemente, a equipe começou os trabalhos aquisição de dados digitais e processamento para a conversão das informações em dados geomagnéticos, em mais alta resolução temporal que o atualmente disponível (médias horárias das componentes geomagnéticas).

\section{Agradecimentos}

Este trabalho foi viabilizado pelas bolsas de estágio concedidas pelo Centro de Integração Empresa-Escola do Rio de Janeiro (CIEE-RJ), bem como pelo Programa de Capacitação Institucional do MCTIC. As atividades foram parcialmente subsidiadas pelas agências de fomento à pesquisa CNPq (projeto 165161/2018-3) e FAPERJ (projeto E-26/203.302/2017). 